\title{
Im Text verwendete sprachliche Abkürzungen
}

\begin{tabular}{|c|c|c|c|}
\hline Abk. & Abkürzung(en) & i.c. & intrakutan \\
\hline Ag & Antigen(e) & IE & Internationale Einheit(en) \\
\hline Aggl. & Agglutination & i.d. & intradermal \\
\hline $\mathrm{Ak}$ & Antikörper. & i.m: & intramuskulär \\
\hline allg. & allgemein & insbes. & insbesondere \\
\hline ausschl. & ausschließlich & i.p. & intraperitoneal \\
\hline bzw. & beziehungsweise & IR & Infrarot \\
\hline Bakt. & Bakterien & i.tr. & intratracheal \\
\hline bakt. & bakteriell & i.v. & intravenös \\
\hline beob. & beobachtet & Immunol. & Immunologie \\
\hline bes. & besonders & immunol. & immunologisch \\
\hline best. & bestehen & klin. & klinisch \\
\hline biol. & biologisch & $\mathbf{L}$ & Literatur \\
\hline ea. & (circa), ungefähr & $\min$. & Minute \\
\hline chron. & chronisch & modif. & modifiziert \\
\hline D & Definition & o.g. & obengenannt \\
\hline d & dies, Tag(e) & $\mathbf{P}$ & Praxis \\
\hline DD & Differentialdiagnose & Pat. & Patient \\
\hline dd & differentialdiagnostisch & $\mathbf{S}$ & Synonyma \\
\hline dgl. & desgleichen & sec. & Sekunde \\
\hline d. h. & das heißt & s.c. & subcutan \\
\hline Di & Diagnose(n), Diagnostik & Serol. & Serologie \\
\hline di & diagnostisch & serol. & serologisch \\
\hline einschl. & einschließlich & sog. & sogenannte \\
\hline entspr. & entsprechend & s. u. & siehe unten \\
\hline et al. & und andere & Syn. & synonym \\
\hline evtl. & eventuell & $\mathrm{Tb}$ & Tuberkulose \\
\hline G & Grundlage(n) & Th & Therapie \\
\hline ggfls. & gegebenenfalls & th & therapeutisch \\
\hline $\mathbf{H}$ & Historie & u. ä., u. a. & sw., vgl., z. B., z. T., z. Z. \\
\hline $\mathbf{h}$ & hora, Stunde(n) & UV & Ultraviolett \\
\hline i.a. & intraarteriell & Vol. & Volumen \\
\hline
\end{tabular}

